\title{
Stress and Health Habits in College Students
}

\author{
Gretchen E. Fogle, Terry F. Pettijohn \\ Department of Psychology, The Ohio State University, Marion, USA \\ Email: Pettijohn.1@osu.edu
}

Received October 31, 2012; revised November 3, 2012; accepted November 10, 2012

Copyright (C) 2013 Gretchen E. Fogle, Terry F. Pettijohn. This is an open access article distributed under the Creative Commons Attribution License, which permits unrestricted use, distribution, and reproduction in any medium, provided the original work is properly cited.

\begin{abstract}
Research has demonstrated that college students experience stress from sources such as poor self-care habits, educational demands, daily hassles, and perceived control over situations. The present study examined perceived stress, health habits, and daily hassles and uplifts among 135 college freshmen. We hypothesized that students with lower stress levels would be male, would have better self-care health habits, would experience fewer minor medical health issues, would have higher academic performance, and would experience fewer daily hassles and more daily uplifts than students who experienced high perceived stress. Strong support was obtained for the hypothesis that students with low perceived stress had better health habits. Students with low perceived stress also experienced significantly fewer hassles and more uplifts per month. There were no significant effects of perceived stress on grade point average or minor medical issues, and there were no significant gender differences in levels of stress. The results could help college freshmen adjust to challenges of college by helping them understand some of the effects of stress and benefits of reducing that stress.
\end{abstract}

Keywords: Stress; Health Habits; College Students; Personality

\section{Introduction}

College students often experience stress from a variety of sources, including poor self-care habits, educational demands, daily hassles, and perceived control over stressful situations. These variables can affect students' academic performance and overall well-being. A number of studies have provided insight into stress and various aspects of health in college students.

Previous research has tended to focus on one aspect of stress or one type of health behavior. We were interested in the role that perceived stress level played in health habits in college students, and thus we extend previous research by examining several hypotheses at the same time. We hypothesized that stress levels in college students would affect self-care health habits, minor medical health issues, academic performance, and overall daily hassles and daily uplifts. It is important to understand the complex issues of stress and health, with a main focus on college students, and an eye on what might be done to help future students.

Garrard and Brumby [1] demonstrated that students perceive stress in a variety of ways. While the majority of students surveyed reported negative associations with stress, $15 \%$ of the college students reported that having a small amount of stress in one's life was beneficial, and $20 \%$ of high school students reported having no stress other than small, everyday hassles.

Ragsdale, Beehr, Grebner, and Han [2] hypothesized that students experience a period of stress during the week due to academic demands, as well as a period of weekend recovery that occurs while performing relaxing, enjoyable activities. Students who engaged in weekend recovery activities rather than using weekends to perform more stressful tasks such as homework reported better wellbeing on Mondays.

Sleep and stress in students was studied by Lund, Reider, Whiting, and Prichard [3]. Their results suggested that perceived stress was related to poor sleep quality. In fact, perceived stress ranked higher than caffeine, alcohol, and exercise in predicting decreased quality of a participant's sleep.

Ruthig, Marrone, Hladkyj, and Robinson-Epp [4] studied the relationship between health behaviors and academic performance in college students. Women reported greater stress and poorer self-care habits than men at the beginning of the fall semester. Both gender groups reported a decrease in stress as the school year progressed, perhaps from adjusting to the pressures of classes and 
living away from home.

Healthy eating is also related to stress in college students, as reported by Oliver and Wardle [5]. The majority of students who participated in this study, both males and females, dieters and non-dieters, reported snacking behavior under stress and a decrease in their intake of "meal foods." The explanation given for this was that people are more likely to choose energy-dense foods rather than non-energy-dense foods when they are stressed. Roughly half of the students stated that they ate less than usual under stress and the other half stated that they ate more than usual.

DeLongis, Coyne, Dakof, Folkman, and Lazarus [6] studied the relationship of daily hassles and major life events to energy level and the frequency and intensity of somatic symptoms (health issues). They found only a weak relationship between major life events and somatic symptoms. Daily hassles, however, were significantly positively correlated with energy level, and both the frequency and intensity of hassles were correlated with illness. This supported the hypothesis that daily hassles are more positively correlated with stress than major life events are.

Manning and Fusilier [7] studied buffers as a means of lessening negative health symptoms potentially caused by stress. A buffer is defined as a personal characteristic that prevents adverse consequences. Buffers in this study included hardiness, exercise, and social support. Manning and Fusilier discovered that higher levels of hardiness were correlated with lower levels of illness. Additionally, participants with higher levels of social support sought medical attention more often, presumably because they were encouraged to do so by their social support circles.

The factors that buffer the negative effects of stress on physical health were studied by Holahan and Moos [8]. They hypothesized that individuals who experienced less adverse effects of stress would be more self-confident and easy-going, more likely to engage in healthy coping behaviors, and more likely to have high levels of family support. Personality, methods of coping, and family support all correlated significantly with levels of both physical and emotional well-being. In general, men in the stressresistant category reported more easy-going personality characteristics, while women in the stress-resistant category reported more family support.

Von Ah, Ebert, Ngamvitroj, Park, and Kang [9] examined the impact of perceived stress, availability of and satisfaction with social support, and self-efficacy on health behaviors in college students. Self-efficacy was the strongest predictor of good health behaviors. In other words, an individual must believe that a healthy behavior is making a difference in order to keep participating in the behavior.
Roddenberry and Renk [10] noted self-efficacy and locus of control as mediators of stress, illness, and utilization of student health services in college students. They found a relationship between a higher level of general self-efficacy and greater overall health, as well as a relationship between a high level of academic self-efficacy and a high level of psychological well-being. In general those who had high levels of stress also had low selfefficacy, high external locus of control, and high levels of illness.

\section{Current Study Hypotheses}

In the present study, we examined five hypotheses on stress and self-care health habits in first-year college students.

Hypothesis 1 stated that male students would experience less stress than female students. While both genders experience stress, females have to deal with a unique set of societal and personal expectations that might cause them to experience more stress.

Hypothesis 2 stated that students who experienced low levels of perceived stress would have better self-care habits than those who experience high levels of perceived stress. Students who have taken care of their basic needs should be equipped to handle stress better.

Hypothesis 3 stated that students with lower stress levels would experience fewer minor medical health issues. The reasoning behind this is that the body will have more energy to expend on everyday activities when it is not busy reacting to stress.

Hypothesis 4 stated that students with lower stress levels would report significantly higher academic performance. These students might be better at coping with stress and at not allowing it to affect their schoolwork.

Hypothesis 5 stated that students with low stress levels would experience fewer daily hassles and more daily uplifts. Daily hassles can negatively affect a student's focus and attitude, while daily uplifts may improve his or her overall mood and performance.

\section{Method}

\subsection{Participants}

One hundred thirty-five undergraduate college students who were taking an introductory psychology course served as participants in this study. The participants were asked to complete an online survey that measured their perceived stress level, self-care health habits, and personality variables related to stress and health. Some students did not answer all questions for some of the scales, and thus were excluded from that particular analysis.

Seventy-five $(55.6 \%)$ of the participants identified themselves as female and $60(44.4 \%)$ identified themselves as male. Ninety-one (67.4\%) students identified 
themselves as freshmen, $33(24.4 \%)$ identified themselves as sophomores, $3(2.2 \%)$ identified themselves as juniors, $2(1.5 \%)$ identified themselves as seniors, and 6 $(4.4 \%)$ identified themselves as another rank.

The mean age of the participants was 20.02 years. The majority of the students were between the ages of 18 and 20 years. The range was $18-47$ years. Fifty-nine (44.4\%) of the participants were 18 years old, $30(22.6 \%)$ were 19 years old, and $19(14.3 \%)$ were 20 years old. $23(17.0 \%)$ of the participants were African American, and 96 (71.1\%) of the participants were Caucasian. In addition, $2(1.5 \%)$ identified themselves as Latin American, 5 (3.7\%) as Asian American, $2(1.5 \%)$ as Native American, and 13 $(9.6 \%)$ as another ethnicity.

$86(65.2 \%)$ of participants identified themselves as single, $29(21.5 \%)$ identified themselves as being in a long-term relationship, $6(4.4 \%)$ identified themselves as engaged, $0(0 \%)$ identified themselves as divorced, 1 $(0.7 \%)$ identified himself or herself as separated, and 6 $(4.4 \%)$ identified themselves as being partnered.

\subsection{Measures}

A variety of scales were used to measure participants' stress, health habits, and personality characteristics. Participants were asked to select one answer for each question.

Overall stress was determined by Cohen, Kamarck and Mermelstein's [11] 14-item Perceived Stress Scale (PSS), developed to provide an objective measurement of individuals' perceptions of stressful events that had occurred in the past month and the intensity of the consequences. The response format was a numerical scale of zero (never) to four (very often). The PSS has both high internal validity and test-retest consistency.

Participants' engagement in health habits was measured by an 18-item scale we created for this study. It was divided into three sections: eating habits, exercise habits, and time management and relaxation, with a numerical response format ranging from zero (never) to four (very often). A self-report measure of the frequency and intensity of any minor medical symptoms the participant had experienced in the past six months was also included. The four response options for symptom frequency were not at all, rarely, sometimes, and often. The three response options for symptom intensity were mild, moderate, and intense.

Participants were given Wallston, Wallston, and DeVellis' [12] 18-item health locus of control scale, the Multidimensional Health Locus of Control Scale (MHLOC), which was created in order to measure attributions for physical health and illness. The MHLOC measures the sources of reinforcement for health related behaviors, including internal (I) sources, chance (C) variables, and being under the control of powerful others $(\mathrm{P})$. The re- sponse format was numerical and ranged from one (strongly disagree) to six (strongly agree).

Participants were also given Gosling, Rentfrow, and Swann's [13] very brief big five personality scale, the Ten Item Personality Inventory (TIPI). The TIPI was developed in response to a need for a less time-consuming alternative to the original big five personality scales used to measure traits of emotional stability, extraversion, openness, agreeableness, and conscientiousness. They found that the briefer scale maintained acceptable accuracy and validity. Unlike the long scale, which focuses on an individual's preferences, the short scale outright asks whether or not the person possesses certain traits. The extent to which the person possesses those traits is rated on a numerical scale of one (disagree strongly) to seven (agree strongly).

Kanner, Coyne, Schaefer, and Lazarus [14] developed the Daily Hassles and Uplifts Scales to measure daily hassles and uplifts. Hassles are defined as annoyances that are not major but still have an effect on a person's daily life. The opposite of hassles are uplifts, defined as small, pleasurable events that make one's day appear better. Hassles and uplifts work together to affect a person's mood and attitudes. Participants rated a list of items as hassles on a scale from zero (item is not a hassle at all) to four (item is a great deal of a hassle). They then rated the list of items as uplifts on a scale of zero (item is not an uplift at all) to four (item is a great deal of an uplift). The same list was used to rate both hassles and uplifts because a person might see some of the items (for example, money) as both a hassle and an uplift.

\subsection{Procedure}

The study was conducted at the Ohio State University Marion Campus during Autumn 2011. The 135 participants took an anonymous online survey and were asked to provide honest responses to the questions. They received research credit that partially fulfilled a requirement for their introductory psychology course. Students had choices of research participation, and the participants freely selected our survey. The study was approved by the Ohio State University IRB, and participants were given the option to withdraw from the study at any time with no penalty, and to skip questions they preferred to not answer. No individual identifying data were collected for this research.

\section{Results}

All of the data presented include means and standard deviations (SD), and for each t-test we included a measure of effect size, the Cohen's d. In all cases of significance, the Cohen's d statistic indicated that the finding had a large effect. 
The Perceived Stress Scale [11] was used to obtain a measure of stress experienced by students. A high stress group of 43 students with a mean perceived stress score of 34.32 ( $\mathrm{SD}=4.30)$ was created from the top one-third of students' scores on the perceived stress scale, and a low stress group of 44 students with a mean perceived stress score of 18.60 ( $\mathrm{SD}=4.52)$ was created from the bottom one-third of students' scores. A t-test for the scores of the high perceived stress students and the low perceived stress students showed this split produced a highly significant difference, $\mathrm{t}(85)=-16.62, \mathrm{p}<0.001, \mathrm{~d}$ $=3.61$. The low stress group scores ranged from 7 to 24 , and the high stress group scores ranged from 30 to 48 . PSS scores could range from 0 to 56 . These two groups were used to test hypotheses on high versus low perceived stress in students.

\subsection{Hypothesis 1: Perceived Stress and Gender}

The first hypothesis stated that males would experience less perceived stress than females. This might be due to environmental conditioning and different societal pressures between genders. Hypothesis 1 was tested with $\mathrm{N}=$ 121 participants (57 males and 64 females). The results of the t-test on the Perceived Stress Scale and gender, $\mathrm{t}$ $(119)=1.52, \mathrm{p}=0.13, \mathrm{~d}=0.28$, did not support this hypothesis. The mean perceived stress score overall was 26.63 (SD = 7.69). Females did experience a slightly higher level of stress than males. The mean perceived stress score for females was $27.63(\mathrm{SD}=7.66)$ and the mean perceived stress score for males was 25.51 ( $\mathrm{SD}=$ 7.62). Although these results were not significant, they did show a slight trend in the direction of the hypothesis.

Results for the Ten Item Personality Inventory (TIPI) scale found that perceived stress had a significant effect on the personality dimension of emotional stability, but not on extraversion, openness, agreeableness, or conscientiousness. Participants could score between 1 and 7 on each dimension. $\mathrm{N}=85$ participants were used to examine effects of perceived stress on emotional stability. Results showed a significant effect of perceived stress on emotional stability, $\mathrm{t}(83)=4.97, \mathrm{p}<0.001, \mathrm{~d}=1.09$. The mean emotional stability score for low stress participants was $5.30(\mathrm{SD}=1.06)$, and the mean emotional stability score for high stress participants was $3.99(\mathrm{SD}=1.35)$. Results indicated that students with low perceived stress were significantly more emotionally stable, and showed less negative emotion.

Males scored significantly higher than females on emotional stability, $\mathrm{t}(128)=-3.36, \mathrm{p}<0.001, \mathrm{~d}=0.59$. The mean TIPI emotional stability score for males was $5.08(\mathrm{SD}=1.13)$ and the mean emotional stability score for females was $4.34(\mathrm{SD}=1.34)$. Males may have a greater overall emotional stability than females, but it is also possible that men reported less experience of negative emotions than females did.

Females scored significantly higher than males on agreeableness, $\mathrm{t}(128)=1.97, \mathrm{p}=0.05, \mathrm{~d}=0.35$. The mean TIPI agreeableness score for females was $5.03(\mathrm{SD}=1.09)$ and the mean agreeableness score for males was 4.68 $(\mathrm{SD}=0.90)$. Females may report higher agreeableness due to socialization. While dominance and aggression are generally acceptable in males, agreeableness is viewed as a more desirable trait in females.

\subsection{Hypothesis 2: Perceived Stress and Health Habits}

The second hypothesis stated that participants who scored low on the Perceived Stress Scale would have better nutrition habits, exercise habits, and time management skills. Six health habits were included in each category. The score for each health habit subscale (nutrition, exercise, and time management) could range from 6 to 30, resulting in an overall score of between 18 and $90 . \mathrm{N}=82$ participants were used to test Hypothesis 2.

Results provided support for this hypothesis. The effect of perceived stress level (high or low) on overall health behaviors was tested. The result for overall health behaviors was significant, $\mathrm{t}(80)=3.89, \mathrm{p}<0.01, \mathrm{~d}=0.87$. The mean score for health behaviors was 61.38 (SD = 9.10) in students who perceived low stress and 53.35 (SD $=9.62$ ) in students who perceived high stress, indicating that low stress students had better overall health care habits.

Individual scoring of each health behavior category further demonstrates that low stress students consistently show better health behaviors across all categories measured. $\mathrm{N}=85$ participants were used to test perceived stress and nutrition habits. The result for nutrition habits was significant, $\mathrm{t}(83)=3.29, \mathrm{p}<0.01, \mathrm{~d}=0.72$. The mean nutrition score was $21.24(\mathrm{SD}=3.40)$ for low stress students and 18.65 ( $\mathrm{SD}=3.84)$ for high stress students, indicating that low stress students had better nutrition habits.

$\mathrm{N}=84$ participants were used to test exercise habits and perceived stress. The result for exercise was significant, $\mathrm{t}(82)=3.01, \mathrm{p}<0.01, \mathrm{~d}=0.66$. The mean exercise score was $19.14(\mathrm{SD}=5.16)$ for low stress students and $15.48(\mathrm{SD}=5.97)$ for high stress students, indicating that low stress students had better exercise habits.

$\mathrm{N}=85$ participants were used to test time management and perceived stress. The result for time management was significant, $\mathrm{t}(83)=3.14, \mathrm{p}<0.01, \mathrm{~d}=0.69$. The mean time management score was $21.00(\mathrm{SD}=3.36)$ for low stress students and 18.79 (SD = 3.13) for high stress students, indicating that low stress students had better time management habits. 
Fifty-nine (44.0\%) students reported sleeping 6 or less hours in an average night, and 75 students $(56.0 \%)$ reported sleeping between 7 - 9 hours per night. Among participants who reported 6 or less hours of sleep per night, $36.7 \%$ perceived low stress while $63.3 \%$ perceived high stress. Among participants who reported 7 - 9 hours of sleep per night, $56.1 \%$ perceived low stress while $43.9 \%$ perceived high stress. One hundred thirty-four participants were used to measure sleep habits with the result that $\chi^{2}(1)=2.98, p=0.08$. There was not a significant difference on perceived stress and sleep.

The effect of gender on health habits was also analyzed. The test for gender and health habits, $\mathrm{t}(123)=-1.19$, $\mathrm{p}=0.24, \mathrm{~d}=0.21$, was not significant. The mean score for females on overall health behaviors was $58.01(\mathrm{SD}=$ 10.64) and the mean score for males on overall health behaviors was $60.18(\mathrm{SD}=9.41)$. The test for gender and nutrition, $\mathrm{t}(129)=-1.19, \mathrm{p}=0.24, \mathrm{~d}=0.21$, was not significant. The mean nutrition score for females was 20.01 $(\mathrm{SD}=4.23)$ and the mean nutrition score for males was 20.83 (SD = 3.43). Exercise habits and gender fell just short of significance, $\mathrm{t}(126)=-1.93, \mathrm{p}=0.06, \mathrm{~d}=0.34$. The mean exercise score for females was $17.34(\mathrm{SD}=$ 5.52) and the mean exercise score for males was 19.30 $(\mathrm{SD}=5.96)$. The trend of males exercising more than females could be due to males being conditioned to consider exercising as more sex-role appropriate. The test for time management and gender, $\mathrm{t}(128)=0.51, \mathrm{p}=0.61, \mathrm{~d}$ $=0.09$, was not significant. The mean time management score for females was $20.25(\mathrm{SD}=3.69)$ and the mean time management score for males was $19.97(\mathrm{SD}=2.53)$.

$\mathrm{N}=132$ students were used to examine exercise habits. Results showed significant differences in exercise habits, $\chi^{2}(3)=22.91, p<0.001$. Thirty-six students $(27.1 \%)$ participated in less than one hour of exercise per week, 54 (40.6\%) participated in 1 - 3 hours per week, 18 (13.5\%) participated in 4 - 5 hours per week, and 24 (18.5\%) participated in 6 or more hours per week.

$\mathrm{N}=133$ participants were used to examine nutrition habits. Results showed significant differences in reported nutrition habits, $\chi^{2}(3)=63.66, p<0.001$. Thirty-two (24.1\%) students stated that they often considered their eating habits nutritious, while 69 (51.9\%) sometimes considered their eating habits nutritious. Additionally, 27 $(20.3 \%)$ rarely considered their eating habits nutritious and $5(3.8 \%)$ never considered their eating habits to be nutritious.

$\mathrm{N}=132$ participants were surveyed on their perceptions of their physical health. 68 (50.7\%) students reported usually considering themselves physically healthy, 51 (38.1\%) sometimes considered themselves physically healthy and $15(11.2 \%)$ rarely considered themselves to be physically healthy.

For Wallston, Wallston, and DeVellis' [12] Multidi- mensional Health Locus of Control Scale (MHLOC), there was a significant difference on the internal subscale for gender, $\mathrm{t}(123)=-2.30, \mathrm{p}=0.02, \mathrm{~d}=0.42$. Males scored more internal than females, $27.2(\mathrm{SD}=3.69)$ and $25.5(\mathrm{SD}=4.65)$, respectively. There were no gender differences for the chance or powerful others subscales.

Overall, the current data for the internal multidimensional health locus of control is similar to what Wallston and colleagues found. Current students' overall mean for internal multidimensional health locus of control was 26.26 , which is very similar to the mean of 25.10 that Wallston and colleagues found for their sample.

\subsection{Hypothesis 3: Perceived Stress and Minor Medical Issues}

The third hypothesis was that participants with a low perceived stress score would experience fewer minor medical issues. The results, $\mathrm{t}(85)=-0.62, \mathrm{p}=0.54, \mathrm{~d}=0.13$, did not support this hypothesis. The mean score for minor medical issues per month was $1.65(\mathrm{SD}=1.23)$ for low stress students and $1.82(\mathrm{SD}=1.28)$ for high stress students.

There was a significant difference between males and females in students' reported minor medical issues. The result for minor medical issues was significant, $\mathrm{t}(131)=$ $2.99, \mathrm{p}<0.01, \mathrm{~d}=0.52$. The mean score for minor medical issues was $2.10(\mathrm{SD}=1.37)$ in females and $1.47(\mathrm{SD}$ $=0.98)$ in males. Thus females reported more minor medical issues than males did.

It is possible that females actually did experience more minor medical issues than males, but it is also possible that males reported fewer issues out of concern for being viewed as weak. Additionally, differences may have existed between males and females regarding what constituted a minor medical issue.

When surveyed about how many times they had experienced a minor medical issue such as a headache, cold, stomachache, or muscle pain unrelated to a chronic condition in the past six months, significant differences were found, $\chi^{2}(4)=151.70, p<0.001 .83(62.4 \%)$ students reported experiencing a minor medical issue 0 - 10 times in the past six months, $18(13.5 \%)$ reported $11-20$ times, $14(10.5 \%)$ reported 21 - 30 times, $10(7.5 \%)$ reported 31 40 times, and $8(6.0 \%)$ reported 41 or more times. 45 (33.3\%) students rated the discomfort caused by these issues as minimal, $56(41.5 \%)$ as minor, $32(23.7 \%)$ as moderate, and $2(1.5 \%)$ as intense.

\subsection{Hypothesis 4: Perceived Stress and Academic Performance}

The fourth hypothesis was that participants with a low perceived stress score would show higher academic performance. We used high school grade point average 
(GPA) as the dependent measure. The grade point average is the mean numerical score of a student's grades, with the highest grade being 4 , and the lowest grade a 0 . The results for the effect of perceived stress on high school grade point average, $\mathrm{t}(84)=1.06, \mathrm{p}=0.29, \mathrm{~d}=$ 0.23 , did not support this hypothesis. Because freshmen college students did not yet have a college grade point average, their high school grade point average was used. The mean reported high school grade point average was $3.34(\mathrm{SD}=0.54)$ for low stress students and $3.22(\mathrm{SD}=$ 0.54 ) for high stress students. While lower stress students had a slightly higher high school grade point average, the difference was not significant.

\subsection{Hypothesis 5: Perceived Stress, Hassles, and Uplifts}

The fifth hypothesis was that people with a low perceived stress score would experience fewer daily hassles and more daily uplifts. Results from the Daily Hassles and Uplifts Scales [14] provided evidence that both hassles and uplifts were significantly affected by the amount of perceived stress a person experienced. Overall, students experienced a mean of $30.26(\mathrm{SD}=13.60)$ hassles per month compared to a mean of $50.51(\mathrm{SD}=19.37)$ uplifts per month. Students reported significantly more uplifts per month than hassles, $\mathrm{t}(84)=9.32, \mathrm{p}=0.01, \mathrm{~d}=2.03$.

An analysis of perceived stress on daily hassles was significant, $\mathrm{t}(63)=-3.00, \mathrm{p}<0.01, \mathrm{~d}=0.76$. The mean number of hassles experienced in one month was 24.62 $(\mathrm{SD}=11.65)$ for low stress students and $33.36(\mathrm{SD}=$ 11.69) for high stress students. These results indicate that low stress students experienced significantly fewer hassles than did high stress students.

The result for the effect of stress on daily uplifts experienced in one month was also significant, $t(65)=2.93$, $\mathrm{p}<0.001, \mathrm{~d}=0.73$. The mean number of daily uplifts experienced in one month was $55.65(\mathrm{SD}=22.95)$ for low stress students and $41.73(\mathrm{SD}=14.93)$ for high stress students. Thus low stress students experienced significantly more daily uplifts compared to high stress students.

No significant sex differences were found for either hassles or uplifts. The mean number of hassles experienced in one month by females was $31.61(\mathrm{SD}=15.52)$ and by males was $28.72(\mathrm{SD}=10.99)$. The mean number of uplifts for females was $51.12(\mathrm{SD}=19.62)$ and the mean for males was $49.82(\mathrm{SD}=19.29)$.

\section{Discussion}

The statistical analyses run on the data demonstrated that there were significant differences in perceived stress in college students and their health habits. There was also a significant effect for perceived stress and daily hassles and uplifts. There was not, however, significance for perceived stress and gender, minor medical issues, or high school grade point average.

Hypothesis 1, which stated that females would experience more perceived stress than males, was not significant. However, females did show a trend in the direction predicted. One possibility for these results is that men and women experience approximately equal amounts of stress but deal with different types of stressors. One limitation of this study is that it only took the amount of stress into account and did not measure the different types of stressors in students' lives. Analysis of the types of stressors in men's and women's lives could provide more detailed information on how stress affects both genders.

Results for analysis of the Ten Item Personality Inventory (TIPI) scale demonstrated that perceived stress had a significant effect on the personality dimension of emotional stability, but not on extraversion, openness, agreeableness, or conscientiousness. The TIPI analysis also showed that males were significantly more emotionally stable, but that females were significantly more agreeable. Originally we hypothesized that stress might influence personality. The finding that low perceived stress was significant only for emotional stability suggests that emotionality might be a key to understanding stress in college students, and perhaps helping students deal with negative emotions might help them also decrease their stress levels.

Hypothesis 2, which stated that low stress students would have better health habits than high stress students, was supported by the data. Good exercise, nutrition, and time management habits typically lead to better physical health and better prioritization, both of which could potentially reduce some stress in students' lives. Educating students about the relationship between lower stress levels and good health habits could lead to improvement in many areas of their lives. Students might be more likely to utilize better time management skills and take better care of their bodies if they understand the positive consequences of doing so. One possibility is to incorporate this information into a course for incoming freshmen. A course that introduces students to college is already required at many universities, and self-care and stress management are both important skills for success throughout college.

Results from the Multidimensional Health Locus of Control scale indicate that males show a slightly more internal locus of control than females. This might suggest that different strategies should be used to encourage good health habits in males and females. For example, perhaps self-improvement might work better for males, whereas social reinforcement might be better used with females.

Hypothesis 3 stated that students who experienced less 
stress would report fewer minor medical issues than students who experienced high stress. This hypothesis was not supported by data. One possibility is that the group of students surveyed consisted primarily of traditional college students in their late teens or early 20s. Because of their age, the students may not have had a large number of health complaints. One limitation of the current research is that it used self-reported responses, and it is certainly possible that the students did not want to report personal medical issues, even if anonymous.

There was a significant gender difference in reports of minor medical issues, with females reporting more issues than males. This could be because females actually did experience more minor health issues, or perhaps males were less comfortable with reporting stress because of the societal pressure on men to be strong. Again, selfreported measures are a limitation in a study such as this one. Roddenberry and Renk [10] reported a significant relationship between stress and illness in a group of primarily female students. This female majority was addressed as a limitation of their study, but this might actually provide insight into a possibility not addressed by the present study. To address this limitation, future research could explore further the relationship between minor medical issues and stress among college women.

Hypothesis 4 stated that students with a lower perceived stress score would perform better academically than people with a higher perceived stress score. Although this hypothesis was not significant, it did show a slight trend in the direction of the initial hypothesis. It is possible that students who experience low levels of stress perform better academically because they are more mentally able to focus on studying and homework. Higher stressed students may have responsibilities that distract them from studying, such as a high number of work hours or dependent children at home. As with Hypothesis 1, this limitation might require that a more concise measurement of types and intensity of stress might be incurporated in future research.

Another potential limitation of this study is that high school grade point average, not college grade point average, was used to determine academic performance since the majority of participants were incoming freshmen who did not yet have a college grade point average. The trend could possibly have been stronger if grade point average had been assessed after students had been in college for several terms. Additionally, the grade point average for all students was relatively high, raising the possibility that it might have been inflated or not accurately reported by the students. On the other hand, it is also possible that some students might prefer higher levels of stress to motivate themselves to do well in school.

Another possibility is that grade point average actually was represented accurately because high school students with high grades are more likely to get into college than those with lower grades. One method of controlling for this possibility would be to test participants after their first term of college so that college grade point average could be used.

Hypothesis 5 stated that a low perceived stress score would result in a low number of daily hassles and a high number of daily uplifts. In other words, students who were less stressed would experience greater numbers of small pleasures and fewer hassles throughout the day. This hypothesis was strongly supported by the results in the current study.

A limitation of the current study was that it did not thoroughly explore what makes an individual perceive a daily event as a hassle or an uplift. Perhaps individuals who experience low stress are more optimistic, thereby perceiving fewer hassles or perhaps viewing hassles as challenges or opportunities rather than threats. Future studies could study the possibility of additional factors being responsible for effects of perceived stress and uplifts and hassles. Future research might also study the effects of increasing awareness of how uplifts and hassles can have an effect in students' lives.

Also, it is very likely that the hassles and uplifts listed in the surveys given to participants were not the only ones they experienced. An opportunity for students to write down other hassles and uplifts not used in the current study could reduce this possibility in future studies.

A better understanding of stress could be beneficial to college students in several ways. First, knowledge of which gender experiences more stress could be helpful in directing specific aid to those who need it most. For example, programs targeted specifically at helping women manage stress may meet their needs better than a general approach. Also, understanding how stress is related to illness and proper self-care health habits has the potential to help students become healthier and thereby better able to handle stress.

Evidence of the importance of good self-care habits may encourage students to refrain from engaging in unhealthy habits. Overall, an awareness of what causes stress may empower students to take appropriate preventative measures.

\section{REFERENCES}

[1] J. Garrard and M. Brumby, "Living and Learning in a Hectic World: Students' Perceptions of Stress," Research in Science Education, Vol. 15, No. 1, 1985, pp. 58-67. doi:10.1007/BF02356526

[2] J. M. Ragsdale, T. A. Beehr, S. Grebner and K. Han, “An Integrated Model of Weekday Stress and Weekend Recovery of Students," International Journal of Stress Management, Vol. 18, No. 2, 2011, pp. 153-180. doi: $10.1037 / \mathrm{a} 0023190$ 
[3] H. G. Lund, B. D. Reider, A. B. Whiting and J. R. Prichard, "Sleep Patterns and Predictors of Disturbed Sleep in a Large Population of College Students," Journal of Adolescent Health, Vol. 46, No. 2, 2010, pp. 124-132. doi:10.1016/j.jadohealth.2009.06.016

[4] J. C. Ruthig, S. Marrone, S. Hladkyj and N. RobinsonEpp, "Changes in College Student Health: Implications for Academic Performance," Journal of College Student Development, Vol. 52, No. 3, 2011, pp. 307-320. doi:10.1353/csd.2011.0038

[5] G. Oliver and J. Wardle, "Perceived Effects of Stress on Food Choice," Physiology and Behavior, Vol. 66, No. 3, 1999, pp. 511-515. doi:10.1016/S0031-9384(98)00322-9

[6] A. DeLongis, J. C. Coyne, G. Dakof, S. Folkman and R. S. Lazarus, "Relationship of Daily Hassles, Uplifts, and Major Life Events to Health Status," Health Psychology, Vol. 1, No. 2, 1982, pp. 119-136. doi:10.1037/0278-6133.1.2.119

[7] M. R. Manning and M. R. Fusilier, "The Relationship between Stress and Health Care Use: An Investigation of the Buffering Roles of Personality, Social Support, and Exercise," Journal of Psychosomatic Research, Vol. 47, No. 2, 1999, pp. 159-173. doi:10.1016/S0022-3999(99)00028-8

[8] C. J. Holahan and R. H. Moos, "Life Stress and Health: Personality, Coping, and Family Support in Stress Resistance," Journal of Personality and Social Psychology, Vol. 49, No. 3, 1985, pp. 739-747. doi:10.1037/0022-3514.49.3.739

[9] D. Von Ah, S. Ebert, A. Ngamvitroj, N. Park and D. Kang, "Predictors of Health Behaviours in College Students," Journal of Advanced Nursing, Vol. 48, No. 5, 2004, pp. 463-474. doi:10.1111/j.1365-2648.2004.03229.x

[10] A. Roddenberry and K. Renk, "Locus of Control and Self-Efficacy: Potential Mediators of Stress, Illness, and Utilization of Health Services in College Students," Child Psychiatry and Human Development, Vol. 41, No. 4, 2010, pp. 353-370. doi:10.1007/s10578-010-0173-6

[11] S. Cohen, T. Kamarck and R. Mermelstein, "A Global Measure of Perceived Stress," Journal of Health and Social Behavior, Vol. 24, No. 4, 1983, pp. 385-396. doi: $10.2307 / 2136404$

[12] K. A. Wallston, B. S. Wallston and R. DeVellis, "Development of the Multidimensional Health Locus of Control Scales," Health Education Monographs, Vol. 6, No. 1, 1978, pp. 160-170. doi:10.1177/109019817800600107

[13] S. D. Gosling, P. J. Rentfrow and W. B. Swann Jr., "A Very Brief Measure of the Big-Five Personality Domains," Journal of Research in Personality, Vol. 37, No. 6, 2003, pp. 504-528. doi:10.1016/S0092-6566(03)00046-1

[14] A. D. Kanner, J. C. Coyne, C. Schaefer and R. S. Lazarus, "Comparison of Two Modes of Stress Measurement: Daily Hassles and Uplifts Versus Major Life Events," Journal of Behavioral Medicine, Vol. 4, No. 1, 1981, pp. 1-39. doi:10.1007/BF00844845 